\title{
Eine mutige alte Dame auf dem goldenen Weg
}

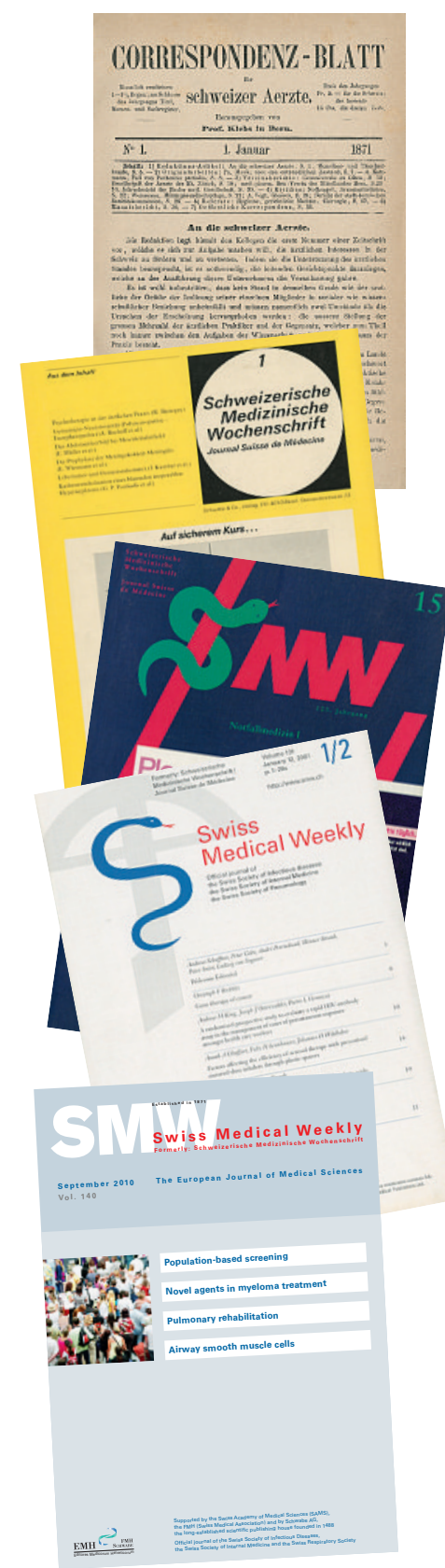

Erinnern Sie sich noch an die «SchwiWo»? Dies war der liebevolle Spitzname der altehrwürdigen «Schweizer Medizinischen Wochenschrift». Das erste Heft erschien 1871 - diese Zeitschrift hat die Entwicklung moderner Publikationsformen wirklich von Anfang an miterlebt. Sie hat sich angepasst und sich immer wieder neu erfunden. Zunehmend schwierig wurde aber, gleichzeitig praxisbezogene Fortbildungsinhalte zu publizieren und auch ein Publikationsorgan für Forschung zu sein, das versuchte, einen respektablen Impact factor zu erreichen. Vor fast 10 Jahren wurde die «SchwiWo» daher aufgeteilt in zwei neue Zeitschriften. Die eine, das «Schweizer Medizin-Forum», ist inzwischen die meistgelesene Fortbildungszeitschrift der Schweiz.

Die andere, das englischsprachige «Swiss Medical Weekly», schickte sich an, die lange Tradition als Plattform insbesondere auch für den wissenschaftlichen Nachwuchs der Schweiz auf hohem Niveau weiterzuführen. Mit Erfolg: Der Impact factor stieg von früher 0,3 auf aktuell 1,681 an und steigt weiter!

Und jetzt dies: SMW wurde kürzlich zu einer primären Online-Zeitschrift mit einer monatlichen Printausgabe. Warum? Das Internet hat wissenschaftliche Publikationsformen komplett umgekrempelt. SMW hatte schon zu Pionierzeiten eine eigene Website lanciert und sich sehr früh zu «open access» bekannt. Denn wir sehen in «open access» nicht eine Gefahr für die Verleger, sondern eine Chance für die Verbreitung von Wissen. Open access bietet für Forschungsresultate eine früher undenkbare globale Verfügbarkeit. Das neue Publikationsmodell der «continuous online publication» ist nun eine weitere Verbesserung: Es beschleunigt die Publikation neuer Forschungsergebnisse enorm, und die umständliche «e-publication ahead of print» fällt komplett weg.

In der Open-access-Bewegung wird die OA-Publikation in einer wissenschaftlichen Zeitschrift übrigens als «goldener Weg» bezeichnet [1]. Denn eine Verlagszeitschrift profitiert von der Sorgfalt einer wissenschaftlichen Redaktion und von einem komplexen Qualitätsprozess. Die Qualität der Beiträge im SMW wird durch internationales Peer reviewing garantiert. Für die Prüfung statistischer Auswertungen arbeiten wir mit renommierten Statistikern zusammen. Mediziner und Übersetzer, alle englischer Muttersprache, lektorieren alle Beiträge vor der Publikation. Schliesslich werden die Daten von Fachleuten in der Medienvorstufe aufbereitet, typographisch korrigiert, für Print und Web gestaltet und publiziert. Zuletzt laden wir die Summaries neuer Artikel direkt in die Medline.

Und die Leser? SMW richtet sich an alle wissenschaftlich interessierten Klinikerinnen und Kliniker!
Und bei aller Begeisterung für moderne Publikationsformen vergessen wir nicht das Vergnügen, in einer gedruckten Zeitschrift zu blättern. Daher erscheint monatlich eine Printversion des SMW. Darin sind die Resultate aller Originalarbeiten in Form von «Extended abstracts» zusammengefasst. Sie beschreiben auf einer Seite die jeweiligen Forschungsresultate. Alle Details dazu sind auf der neu gestalteten SMW-Website in der ausführlichen Originalpublikation zu finden. Für die Entwicklung dieses neuen Publikationsmodells haben uns die Bedürfnisse der Leserinnen und Leser und der Autorinnen und Autoren geleitet, aber auch herausragende Vorbilder, und wir sind der Chefredaktion des «British Medical Journal» sehr verbunden für die Erlaubnis, für die «Extended abstracts» auf ihre exzellenten Formulare zurückgreifen zu dürfen.

Noch lesenswerter werden die monatlichen Printausgaben des SMW durch die darin publizierten «State of the art»-Reviews: klassische Übersichtsarbeiten und die spannenden Rubriken «Medical intelligence», «Current opinion» und «Medtech».

Ein herausforderndes Projekt wie dieses wäre für einen Verlag nicht möglich ohne Redaktoren, die mit uns an ein Produkt glauben. Wir danken der SMWRedaktion sehr herzlich dafür, dass sie ihr Wissen, ihre Zeit und ihre Kreativität mit uns teilt. Unser gemeinsamer Dank gebührt aber natürlich den Autoren und den vielen Reviewern, die das «Swiss Medical Weekly» erst möglich machen.

Zusammen mit der Schweizerischen Ärztezeitung (Standes- und Gesundheitspolitik) und dem Schweizerischen Medizin-Forum (Weiter- und Fortbildung) gehört SMW zum Drei-Säulen-Modell des publizistischen Auftrags, den der Schweizerische Ärzteverlag EMH erfüllt. Wenn Ihnen die dritte Säule «Forschung» nicht gleichgültig ist, klicken Sie doch einfach mal auf www.smw.ch.

Natalie Marty, Publizistische Leiterin des Schweizerischen Ärzteverlags EMH

PS: Allen Leserinnen und Lesern der «Schweizerischen Ärztezeitung» senden wir gerne eine Probenummer der nächsten Printausgabe des SMW zu. Bestellen Sie Ihr kostenfreies Ansichtsexemplar unter 0614678578 oder per Mail: auslieferung@emh.ch. Viel Spass beim Lesen und Entdecken des neuen traditionsreichen «Swiss Medical Weekly».

1 Lesenswert dazu: http://open-access.net/de/ allgemeines/was_bedeutet_open_access/ 